\title{
Analytic Solutions to Optimal Control Problems with Constraints
}

\author{
Dan Wu \\ Department of Mathematics, Henan University of Science and Technology, Luoyang, China \\ Email: lywd2964@126.com
}

Received 25 November 2015; accepted 28 December 2015; published 31 December 2015

Copyright (C) 2015 by author and Scientific Research Publishing Inc.

This work is licensed under the Creative Commons Attribution International License (CC BY). http://creativecommons.org/licenses/by/4.0/

c) (i) Open Access

\begin{abstract}
In this paper, the analytic solutions to constrained optimal control problems are considered. A novel approach based on canonical duality theory is developed to derive the analytic solution of this problem by reformulating a constrained optimal control problem into a global optimization problem. A differential flow is presented to deduce some optimality conditions for solving global optimizations, which can be considered as an extension and a supplement of the previous results in canonical duality theory. Some examples are given to illustrate the applicability of our results.
\end{abstract}

\section{Keywords}

\section{Constrained Optimal Control, Analytic Solution, Canonical Duality Theory, Global Optimization}

\section{Introduction}

In this paper, we consider the following linear-quadratic optimal control problem involving control constraints:

$$
\begin{aligned}
& (\mathcal{P}) \quad \min \quad J(x, u)=\frac{1}{2} \int_{t_{0}}^{t_{f}} x^{\mathrm{T}}(t) Q x(t)+u^{\mathrm{T}}(t) R u(t) \mathrm{d} t \\
& \\
& \text { s.t. } \quad \dot{x}(t)=A x(t)+B u(t), \quad x\left(t_{0}\right)=x_{0}, \quad u(t) \in U, \quad t \in\left[t_{0}, t_{f}\right],
\end{aligned}
$$

where $Q \in \mathcal{R}^{n \times n}$ is a positive semidefinite symmetric matrix, $R \in \mathcal{R}^{m \times m}$ is a positive definite symmetric matrix, and $A \in \mathcal{R}^{n \times n}, B \in \mathcal{R}^{n \times m}$ are two given matrices. $x(t) \in \mathcal{R}^{n}$ is a state vector, and $u(t) \in \mathcal{R}^{m}$ is an admissible control taking values on the set $U$, which is integrable or piecewise continuous on $\left[t_{0}, t_{f}\right]$. In our work, we suppose that $U$ is a closed convex set, and we study two forms of the set $U$, a sphere constraint and box constraints respectively. Problems of the above type arise naturally in system science and engineering with wide applications [1] [2].

In recent years, significant advances have been made in efficiently tackling optimal control problems [1] [3]. 
In the unconstrained case, an optimal feedback control can be successfully obtained which seems to be a perfect result. For constrained optimal control problems the level of research is less complete. It is now well known that common approaches are based on applying a quasi-Newton or sequential quadratic programming $(S Q P)$ technique to the constrained; see for instance [4]-[8] and the references therein. But due to the presence of state or control constraints, all the above methods are trapped in analytical difficulties and thus are not guaranteed to find analytic solutions to the constrained, at best, they can provide numerical solutions.

In this paper, a different way, canonical dual approach is used to study the problem $(\mathcal{P})$ by converting the original control problem into a global optimization problem. The canonical duality theory was developed from nonconvex analysis and mechanics during the last decade (see [9] [10]), and has shown its potential for global optimization and nonconvex nonsmooth analysis [10]-[14]. Meanwhile, we introduce a differential flow for constructing the so-called canonical dual function to deduce some optimality conditions for solving global optimizations, which is shown to extend some corresponding results in canonical duality theory [9]-[11]. In comparison to the previous work mainly focused on simple constraints, we not only discuss linear box constraints, but also the nonlinear sphere constraint. Then combining the canonical dual approach given in this paper with the Pontryagin maximum principle, we solve the constrained optimal control problem $(\mathcal{P})$ and characterize the analytic solution expressed by the co-state via canonical dual variables.

Now, we shall give the Pontryagin maximum principle and an important Lemma.

Pontryagin Maximum Principle If $\hat{u}(\cdot)$ is an optimal solution to the problem $(\mathcal{P})$ and the corresponding state and co-state are denoted by $\hat{x}(\cdot)$ and $\hat{\lambda}(\cdot)$ respectively, for the Hamilton function

$$
H(t, x, u, \hat{\lambda})=\hat{\lambda}^{\mathrm{T}}(A x+B u)+\frac{1}{2} x^{\mathrm{T}} Q x+\frac{1}{2} u^{\mathrm{T}} R u,
$$

then we have,

$$
\begin{gathered}
\dot{\hat{x}}=H_{\lambda}(t, \hat{x}, \hat{u}, \hat{\lambda})=A \hat{x}+B \hat{u}, \hat{x}\left(t_{0}\right)=x_{0}, \\
\dot{\hat{\lambda}}=-H_{x}(t, \hat{x}, \hat{u}, \hat{\lambda})=-A^{\mathrm{T}} \hat{\lambda}-Q \hat{x}, \hat{\lambda}\left(t_{f}\right)=\overrightarrow{0},
\end{gathered}
$$

and

$$
H(t, \hat{x}(t), \hat{u}(t), \hat{\lambda}(t))=\min _{u \in U} H(t, \hat{x}(t), u(t), \hat{\lambda}(t)) \text {, a.e. } t \in\left[t_{0}, t_{f}\right] .
$$

Lemma 1. An admissible pair $(\hat{x}(t), \hat{u}(t))$ is an optimal pair to the primal problem $(\mathcal{P})$ if and only if this pair $(\hat{x}(t), \hat{u}(t))$ satisfies the Pontryagin maximum principle (3), (4) and (5).

Proof. Denote

$$
H^{*}(t, x, \hat{\lambda})=\min _{u \in U} H(t, x, u, \hat{\lambda}) \text { a.e. } t \in\left[t_{0}, t_{f}\right] .
$$

Let $(x(t), u(t))$ be an arbitrary admissible pair satisfying (3). By the definition of $H^{*}$, we have $H^{*}(t, x, \hat{\lambda}) \leq H(t, x, u, \hat{\lambda})$, and $H^{*}(t, x, \hat{\lambda})$ is equivalent to the following global optimization

$$
\min _{u \in U} \frac{1}{2} u^{\mathrm{T}} R u+\hat{\lambda}^{\mathrm{T}}(t) B u \text {, a.e. } t \in\left[t_{0}, t_{f}\right] .
$$

Moreover, it is easy to see that the minimizer $\hat{u}$ of (7) depends only on the co-state $\hat{\lambda}$, i.e. $\frac{\partial \hat{u}}{\partial x}=0$, which implies that

$$
H_{x}^{*}(t, x, \hat{\lambda})=H_{x}(t, x, \hat{u}, \hat{\lambda})+H_{u}(t, x, \hat{u}, \hat{\lambda}) \frac{\partial \hat{u}}{\partial x}=H_{x}(t, x, \hat{u}, \hat{\lambda}) .
$$

Taking into account of the convexity of the integrand in the cost functional as well as the set $U$, the function $H^{*}(t, x, \hat{\lambda})$ is convex in $x$, and

$$
\begin{aligned}
H^{*}(t, x, \hat{\lambda}) & \geq H^{*}(t, \hat{x}, \hat{\lambda})+H_{x}^{* \mathrm{~T}}(t, \hat{x}, \hat{\lambda})(x-\hat{x}) \\
& =H(t, \hat{x}, \hat{u}, \hat{\lambda})+H_{x}^{\mathrm{T}}(t, \hat{x}, \hat{u}, \hat{\lambda})(x-\hat{x}),
\end{aligned}
$$


which leads to

$$
H(t, \hat{x}, \hat{u}, \hat{\lambda})-H(t, x, u, \hat{\lambda}) \leq \dot{\hat{\lambda}}^{\mathrm{T}}(x-\hat{x}) .
$$

Thus, we have

$$
\begin{aligned}
& J[\hat{u}(\cdot)]-J[u(\cdot)] \\
= & \int_{t_{0}}^{t_{f}}[H(t, \hat{x}(t), \hat{u}(t), \hat{\lambda}(t))-H(t, x(t), u(t), \hat{\lambda}(t))] \mathrm{d} t+\int_{t_{0}}^{t_{f}} \hat{\lambda}^{\mathrm{T}}(t)(\dot{x}(t)-\dot{\hat{x}}(t)) \mathrm{d} t \\
\leq & \int_{t_{0}}^{t_{f}} \mathrm{~d}\left[\hat{\lambda}^{\mathrm{T}}(t)(x(t)-\hat{x}(t))\right]=-\hat{\lambda}^{\mathrm{T}}\left(t_{0}\right)\left(x\left(t_{0}\right)-\hat{x}\left(t_{0}\right)\right)=0 .
\end{aligned}
$$

This means that $J$ attains its minimum at $\hat{u}$. The proof is completed.

The above Lemma reformulates the optimal control problem $(\mathcal{P})$ into a global optimization problem (7). Based on this fact, we can derive the analytic solution of the problem $(\mathcal{P})$ by only solving problem $(7)$ via the canonical dual approach.

The rest of the paper is organized as follows. In Section 2, we consider the optimal control problem with a sphere constraint. By introducing the differential flow and canonical dual function for solving global optimizations, we derive the analytic solution expressed by the co-state via canonical dual variables. Based on the similar canonical dual strategy, the box constrained optimal control problem is studied and the corresponding analytic expression of optimal control is obtained in Section 3. Meanwhile, some examples are given to demonstration.

\section{Sphere Constrained Optimal Control Problem}

In this section, we let $U=\left\{u(t) \in \mathcal{R}^{m} \mid \frac{1}{2} u^{\mathrm{T}}(t) u(t) \leq a, a>0\right\}$ be a sphere. Before we go to derive the analytic solution for the problem $(\mathcal{P})$, we first make some preliminary concepts and theorems in solving global optimization over a sphere based on canonical duality theory which will be used in the sequel.

\subsection{Global Optimization over a Sphere}

Consider the following general optimization problem

$$
\min P(u) \text { s.t. } u \in U, U=\left\{\frac{1}{2} u^{\mathrm{T}} u \leq a\right\},
$$

where $P(u)$ is assumed to be twice continuously differentiable in $\mathcal{R}^{m}$.

The original idea of this section is from the paper [13] by Zhu. Denote

$$
\mathcal{S}=\left\{\rho \in \mathcal{R} \mid\left[\nabla^{2} P(u)+\rho I\right]>\overrightarrow{0}, \rho \geq 0 \text {, for every } u \in U\right\} .
$$

$\mathcal{S}$ is an open set with respect to $[0,+\infty)$, and it is easy to see that if $\hat{\rho} \in \mathcal{S}$, then $\rho \in \mathcal{S}$ for any $\rho \geq \hat{\rho}$.

Assume that a $\rho^{*} \in \mathcal{S}$ and a nonzero vector $u^{*} \in U$ such that

$$
\nabla P\left(u^{*}\right)+\rho^{*} u^{*}=\overrightarrow{0} .
$$

We focus on the differential flow $\hat{u}(\rho)$ which is well defined near $\rho^{*}$ by

$$
\begin{gathered}
\frac{\mathrm{d} \hat{u}}{\mathrm{~d} \rho}+\left[\nabla^{2} P(\hat{u})+\rho I\right]^{-1} \hat{u}=\overrightarrow{0}, \\
\hat{u}\left(\rho^{*}\right)=u^{*} .
\end{gathered}
$$

Based on the classical theory of ODE, we can obtain the solution $\hat{u}(\rho)$ of (12) (13), which can be extended to an interval in $\mathcal{S}$ [2]. Thus, the canonical dual function [9] [10] with respect to a given flow $\hat{u}(\rho)$ is defined as follows 


$$
P^{d}(\rho)=P(\hat{u}(\rho))+\frac{\rho}{2} \hat{u}^{\mathrm{T}}(\rho) \hat{u}(\rho)-\rho a
$$

and the canonical dual problem associated with the problem (10) can be proposed as follows

$$
\left(P^{d}\right): \max \left\{P^{d}(\rho)=P(\hat{u}(\rho))+\frac{\rho}{2} \hat{u}^{\mathrm{T}}(\rho) \hat{u}(\rho)-\rho a \mid \rho \in \mathcal{S}\right\} .
$$

Notice that $\frac{\mathrm{d}^{2} P(\rho)}{\mathrm{d} \rho^{2}}=-\hat{u}^{\mathrm{T}}(\rho)\left[\nabla^{2} P(\hat{u}(\rho))+\rho I\right]^{-1} \hat{u}(\rho)$. By the definition of $\mathcal{S}$, it follows that the canonical dual function $P^{d}(\rho)$ is concave on $\mathcal{S}$. For a critical point $\hat{\rho} \in \mathcal{S}$, it must be a global maximizer of $P^{d}(\rho)$ on $\mathcal{S}$, sometimes, which leads to a global minimizer $\hat{u}=\hat{u}(\hat{\rho})$ of $(10)$.

Theorem 1. If the flow $\hat{u}(\rho)$ (defined by (11)-(13)) meets a boundary point of the ball $U$ at $\hat{\rho} \in \mathcal{S}$ such that $\frac{1}{2} \hat{u}^{\mathrm{T}}(\hat{\rho}) \hat{u}(\hat{\rho})=a$, then $\hat{u}(\hat{\rho})$ is a global minimizer of $P(u)$ over U. Further one has

$$
\min _{u \in U} P(u)=P(\hat{u}(\hat{\rho}))=P^{d}(\hat{\rho})=\max _{\rho \geq \hat{\rho}} P^{d}(\rho) .
$$

Detailed proof of Theorem 1 can be referred to [13]-[15].

In what follows, we show that $\hat{u}(\rho)$ can be derived by solving backward differential equation.

Lemma 2. Let $\hat{u}(\rho)$ be a given flow defined by (11)-(13). We call $\hat{u}(\rho), \rho \in\left(0, \rho^{*}\right]$ a backward differential flow.

Since $U$ is bounded and $P(u)$ is twice continuously differentiable, we can choose a large positive parameter $\rho^{*}$ such that $\left[\nabla^{2} P(u)+\rho^{*} I\right]>0, \forall u \in U$ and $\rho^{*}>\sup _{U}\{\|\nabla P(u)\|\}$. If $\nabla P(0) \neq 0$, then it follows from $\left\|\frac{\nabla^{2} P(u)}{\rho^{*}}\right\|<1$ uniformly in $U$ that there is a unique nonzero fixed point $u^{*} \in U$ such that

$$
\frac{-\nabla P\left(u^{*}\right)}{\rho^{*}}=u^{*}
$$

by Brown fixed-point theorem, which means that the pair $\left(u^{*}, \rho^{*}\right)$ satisfies (11). Then we can solve (11) backwards from $\rho^{*}$ to get the backward flow $\hat{u}(\rho), \rho \in\left(0, \rho^{*}\right]$. We refer the interested reader to [16] [17] for detail of choosing the desired parameter $\rho^{*}$.

\subsection{Analytic Solution to the Sphere Constrained Optimal Control Problem}

Let $P(u)=\frac{1}{2} u^{\mathrm{T}} R u+\hat{\lambda}^{\mathrm{T}}(t) B u$ in (10). Based on the canonical dual approach in Section 2.1, a relationship between $u \in \mathcal{R}^{m}$ and $\rho \in \mathcal{S}=[0,+\infty$ ) (since $R$ is a positive definite matrix) is well defined as

$$
\hat{u}(\rho)=-[R+\rho I]^{-1} B^{\mathrm{T}} \hat{\lambda}(t) \text { a.e. } t \in\left[t_{0}, t_{f}\right] .
$$

So, the canonical dual function can be formulated as, for each $\rho \geq 0$

$$
P^{d}(\rho)=-\frac{1}{2} \hat{\lambda}^{\mathrm{T}}(t) B(R+\rho I)^{-1} B^{\mathrm{T}} \hat{\lambda}(t)-a \rho .
$$

Next, we have the following properties.

Lemma 3. Let $\hat{u}(\rho)$ be a given flow defined by (18) and $y(\rho)=\frac{1}{2} \hat{u}^{\mathrm{T}}(\rho) \hat{u}(\rho)$, we have

$$
\frac{\mathrm{d} P^{d}(\rho)}{\mathrm{d} \rho}=\frac{1}{2} \hat{u}^{\mathrm{T}}(\rho) \hat{u}(\rho)-a=y(\rho)-a,
$$




$$
\frac{\mathrm{d} y(\rho)}{\mathrm{d} \rho}=-\left(\frac{\mathrm{d} \hat{u}(\rho)}{\mathrm{d} \rho}\right)^{\mathrm{T}}[R+\rho I] \frac{\mathrm{d} \hat{u}(\rho)}{\mathrm{d} \rho} .
$$

Proof. Since $P^{d}(\rho)$ is differentiable,

$$
\begin{aligned}
\frac{\mathrm{d} P^{d}(\rho)}{\mathrm{d} \rho} & =-\frac{1}{2} \hat{\lambda}^{\mathrm{T}}(t) B \frac{\mathrm{d}\left[(R+\rho I)^{-1}\right]}{\mathrm{d} \rho} B^{\mathrm{T}} \hat{\lambda}(t)-a \\
& =\frac{1}{2} \hat{\lambda}^{\mathrm{T}}(t) B(R+\rho I)^{-1} \frac{\mathrm{d}(R+\rho I)}{\mathrm{d} \rho}(R+\rho I)^{-1} B^{\mathrm{T}} \hat{\lambda}(t)-a \\
& =\frac{1}{2} \hat{u}^{\mathrm{T}}(\rho) \hat{u}(\rho)-a . \\
\frac{\mathrm{d} y(\rho)}{\mathrm{d} \rho} & =\hat{u}^{\mathrm{T}}(\rho) \frac{\mathrm{d} \hat{u}(\rho)}{\mathrm{d} \rho}=-\left(\frac{\mathrm{d} \hat{u}(\rho)}{\mathrm{d} \rho}\right)^{\mathrm{T}}[R+\rho I] \frac{\mathrm{d} \hat{u}(\rho)}{\mathrm{d} \rho} .
\end{aligned}
$$

Lemma 4. Let $\hat{u}(\rho)$ be a given flow defined by (18), and $P^{d}(\rho)$ be the corresponding canonical dual function defined by (19).

1) $y(\rho)$ is monotonously decreasing on $[0,+\infty)$.

2) if there exists $\hat{\rho} \in[0,+\infty)$ such that $\hat{u}(\hat{\rho}) \in U$, then $P^{d}(\rho)$ is monotonously decreasing on $[\hat{\rho},+\infty)$.

Proof. By (21), it follows that $\frac{\mathrm{d} y(\rho)}{\mathrm{d} \rho} \leq 0$ for any $\rho \in[0,+\infty)$, which means that $y(\rho)$ is monotonously decreasing on $[0,+\infty)$.

If there exists one point $\hat{\rho} \in[0,+\infty)$ and $\hat{u}(\hat{\rho}) \in U$ such that $y(\hat{\rho}) \leq a$, by the monotonous decline of $y(\rho)$, we have $\hat{u}(\rho) \in U$ for any $\rho \geq \hat{\rho}$. By (20), we can conclude that $P^{d}(\rho)$ is monotonously decreasing on $[\hat{\rho},+\infty)$. The proof is completed.

Theorem 2. For the sphere constrained optimal control problem $(\mathcal{P})$, the analytic solution expressed by the co-state is given as follows

$$
\hat{u}=-\left[R+\rho^{o p t}(\hat{\lambda}) I\right]^{-1} B^{\mathrm{T}} \hat{\lambda},
$$

where $\rho^{\text {opt }}(\hat{\lambda})$ with respect to the co-state $\hat{\lambda}$ is defined by the following condition

$$
\rho^{\text {opt }}(\hat{\lambda})= \begin{cases}\hat{\rho}(\hat{\lambda}) & \text { if } \hat{\lambda}^{\mathrm{T}} B R^{-2} B^{\mathrm{T}} \hat{\lambda}>2 a, \\ 0 & \text { if } \hat{\lambda}^{\mathrm{T}} B R^{-2} B^{\mathrm{T}} \hat{\lambda} \leq 2 a,\end{cases}
$$

and $\hat{\rho}(\hat{\lambda})$ satisfies the equation $\hat{\lambda}^{\mathrm{T}} B[R+\rho(\hat{\lambda}) I]^{-2} B^{\mathrm{T}} \hat{\lambda}=2 a, \rho(\hat{\lambda}) \geq 0$.

Proof. We first consider $B^{\mathrm{T}} \hat{\lambda}(t) \neq 0$ for some one point $t \in\left[t_{0}, t_{f}\right]$.

For any $\rho \in[0,+\infty)$, when $B^{\mathrm{T}} \hat{\lambda}(t) \neq 0$, with (12), (18) and taking into account of Lemma 3, we have $\hat{u}(\rho) \neq 0$ and $\frac{\mathrm{d} \hat{u}(\rho)}{\mathrm{d} \rho} \neq 0$. This means that $y(\rho)$ is strictly monotonously decreasing on $[0,+\infty)$.

Case 1: Suppose that $\frac{1}{2} \hat{u}^{\mathrm{T}}(0) \hat{u}(0)>a$. Since $y(\rho)$ is continuous and strictly monotonously decreasing on $[0,+\infty)$ and $y(\rho) \rightarrow 0$ as $\rho \rightarrow+\infty$, there must exist one point $\hat{\rho}>0$ such that $\frac{1}{2} \hat{u}^{\mathrm{T}}(\hat{\rho}) \hat{u}(\hat{\rho})=a$, i.e. $\hat{u}(\hat{\rho}) \in U$, which leads to $\hat{u}(\rho) \in U$ for any $\rho \geq \hat{\rho}$. For each element $\rho, \rho \geq \hat{\rho}$, the function $f_{\rho}(u)$ is given as follows

$$
f_{\rho}(u)=P(u)+\rho\left(\frac{1}{2} u^{\mathrm{T}} u-a\right)=\frac{1}{2} u^{\mathrm{T}} R u+\hat{\lambda}^{\mathrm{T}}(t) B u+\rho\left(\frac{1}{2} u^{\mathrm{T}} u-a\right),
$$


where $\rho$ is a parameter. It is obvious that $P(u) \geq f_{\rho}(u)$ for all $u \in U$. Since $f_{\rho}(u)$ is twice continuously differentiable in $\mathcal{R}^{m}$, there exists a closed convex region $\mathcal{E}$ containing $U$ such that on $\mathcal{E}, \nabla^{2} f_{\rho}(u)>0$ and $\nabla f_{\rho}(\hat{u}(\rho))=0$. This implies that $\hat{u}(\rho)$ is the unique global minimizer of $f_{\rho}(u)$ over $\mathcal{E}$. By (18) and (19), we have

$$
f_{\rho}(\hat{u}(\rho))=-\frac{1}{2} \hat{\lambda}^{\mathrm{T}}(t) B[R+\rho I]^{-1} B^{\mathrm{T}} \hat{\lambda}(t)-\rho a=P^{d}(\rho),
$$

and

$$
P(u) \geq f_{\rho}(u) \geq \min _{u \in U} f_{\rho}(u)=f_{\rho}(\hat{u}(\rho))=P^{d}(\rho) .
$$

Further, it follows from Lemma 4 that

$$
\max _{\rho \geq \hat{\rho}} P^{d}(\rho)=P^{d}(\hat{\rho})=P(\hat{u}(\hat{\rho})) .
$$

Thus, for every $u \in U$, when $\rho \geq \hat{\rho}$, we have

$$
\min _{u \in U} \frac{1}{2} u^{\mathrm{T}} R u+\hat{\lambda}^{\mathrm{T}}(t) B u=\frac{1}{2} \hat{u}^{\mathrm{T}}(\hat{\rho}) R \hat{u}(\hat{\rho})+\hat{\lambda}^{\mathrm{T}}(t) B \hat{u}(\hat{\rho})=P^{d}(\hat{\rho})=\max _{\rho \geq \hat{\rho}} P^{d}(\rho) .
$$

Case 2: Suppose that $\frac{1}{2} \hat{u}^{\mathrm{T}}(0) \hat{u}(0) \leq a$. It is easy to verify that $\frac{1}{2} \hat{u}^{\mathrm{T}}(\rho) \hat{u}(\rho)<a$ for any $\rho>0$, and $\max _{\rho \geq 0} P^{d}(\rho)=P^{d}(0)$. Then, by using the similar proving strategy in case 1 , we can show that $\hat{u}(0)$ is a global minimizer of (7) in case 2.

On the other hand, If there exists one point $t \in\left[t_{0}, t_{f}\right]$ such that $B^{\mathrm{T}} \hat{\lambda}(t)=0$, then (7) is equivalent to the problem $\min _{u \in U} \frac{1}{2} u^{\mathrm{T}} R u$, and it is clear that $\hat{u}(0)=0$ is a global minimizer of this problem.

Define

$$
\rho^{\text {opt }}= \begin{cases}\hat{\rho} & \text { if }\|\hat{u}(0)\|>\sqrt{2 a} \\ 0 & \text { if }\|\hat{u}(0)\| \leq \sqrt{2 a}\end{cases}
$$

where $\hat{\rho}$ is the only solution of the equation $\left\|[R+\rho I]^{-1} B^{\mathrm{T}} \hat{\lambda}(t)\right\|=\sqrt{2 a}, \rho \in[0,+\infty)$ under the condition $\|\hat{u}(0)\|>\sqrt{2 a}$. Based on canonical duality theory, $\hat{u}\left(\rho^{\text {opt }}\right)$ is a global minimizer of the problem (7). Hence, by Lemma 1, we can derive the optimal solution

$$
\hat{u}\left(\rho^{o p t}\right)=-\left[R+\rho^{o p t} I\right]^{-1} B^{\mathrm{T}} \hat{\lambda}(t) \text {, a.e. } t \in\left[t_{0}, t_{f}\right] .
$$

If consider $\rho^{\text {opt }}$ as a function with respect to the co-state $\hat{\lambda}$, we can define the function $\rho^{\text {opt }}(\hat{\lambda})$ satisfying (23), and the analytic solution by the co-state to the problem $(\mathcal{P})$ can be given as (22). This completes the proof.

Theorem 3. Let $R$ be an identity matrix I in (1). Then the analytic solution to problem $(\mathcal{P})$ is obtained as follows

$$
\hat{u}=-\frac{B^{\mathrm{T}} \hat{\lambda}}{\max \left(1, \frac{\left\|B^{\mathrm{T}} \hat{\lambda}\right\|}{\sqrt{2 a}}\right)} .
$$

Proof. Suppose that $R=I$. By Theorem 2, it follows that $\rho^{o p t}=\max \left[0,-1+\frac{\left\|B^{\mathrm{T}} \hat{\lambda}(t)\right\|}{\sqrt{2 a}}\right]$, thus, the analytic 
solution can be expressed as, a.e. $t \in\left[t_{0}, t_{f}\right]$,

$$
\hat{u}\left(\rho^{o p t}\right)=-\left[R+\rho^{o p t} I\right]^{-1} B^{\mathrm{T}} \hat{\lambda}(t)=-\frac{B^{\mathrm{T}} \hat{\lambda}(t)}{\max \left(1, \frac{\left\|B^{\mathrm{T}} \hat{\lambda}(t)\right\|}{\sqrt{2 a}}\right)} .
$$

This concludes the proof of Theorem 3.

\subsection{Applications}

Now, we give an example to illustrate the applicability of Theorem 2. We consider the following sphere constrained optimal control problem.

Example 1: In (1), we consider $A=\left(\begin{array}{ccc}3 & -1 & 2 \\ -5 & 0 & 7 \\ 4 & 4.5 & -7\end{array}\right), \quad B=\left(\begin{array}{cc}3.5 & -2 \\ 2 & 6 \\ 8 & -9\end{array}\right), \quad Q=\left(\begin{array}{ccc}2 & -5 & -7 \\ 1 & 2 & 1.5 \\ -3 & 0 & 8\end{array}\right), \quad R=I_{2 \times 2}$,

$x(0)=(-1,-1,-1)^{\mathrm{T}}, t_{0}=0, t_{f}=1$, and $a=\frac{1}{2} . Q \geq 0$ and $R>0$ satisfy the assumptions in this paper.

By Lemma 1 and Theorem 2, in order to derive the optimal solution of Example 1, we need to solve a system on the state and co-state

$$
\begin{gathered}
\dot{\hat{x}}=H_{\lambda}(t, \hat{x}, \hat{u}, \hat{\lambda})=A \hat{x}+B \hat{u}, \hat{x}(0)=(-1,-1,-1)^{\mathrm{T}}, \\
\dot{\hat{\lambda}}=-H_{x}(t, \hat{x}, \hat{u}, \hat{\lambda})=-A^{\mathrm{T}} \hat{\lambda}-Q \hat{x}, \hat{\lambda}(1)=\overrightarrow{0} .
\end{gathered}
$$

and

$$
\hat{u}=\arg \min _{u^{\mathrm{T}} u \leq 1}\left[\frac{1}{2} u^{\mathrm{T}} u+\hat{\lambda}^{\mathrm{T}} B u\right]=-\frac{B^{\mathrm{T}} \hat{\lambda}}{\max \left[1,\left\|B^{\mathrm{T}} \hat{\lambda}\right\|\right]} \text { a.e. } t \in[0,1] .
$$

By numerical methods of two-point boundary value problems [18] [19], we can obtain the optimal solution $\hat{u}$ and the dual variable $\rho^{\text {opt }}(\hat{\lambda})$ as follows (see Figure 1, Figure 2).

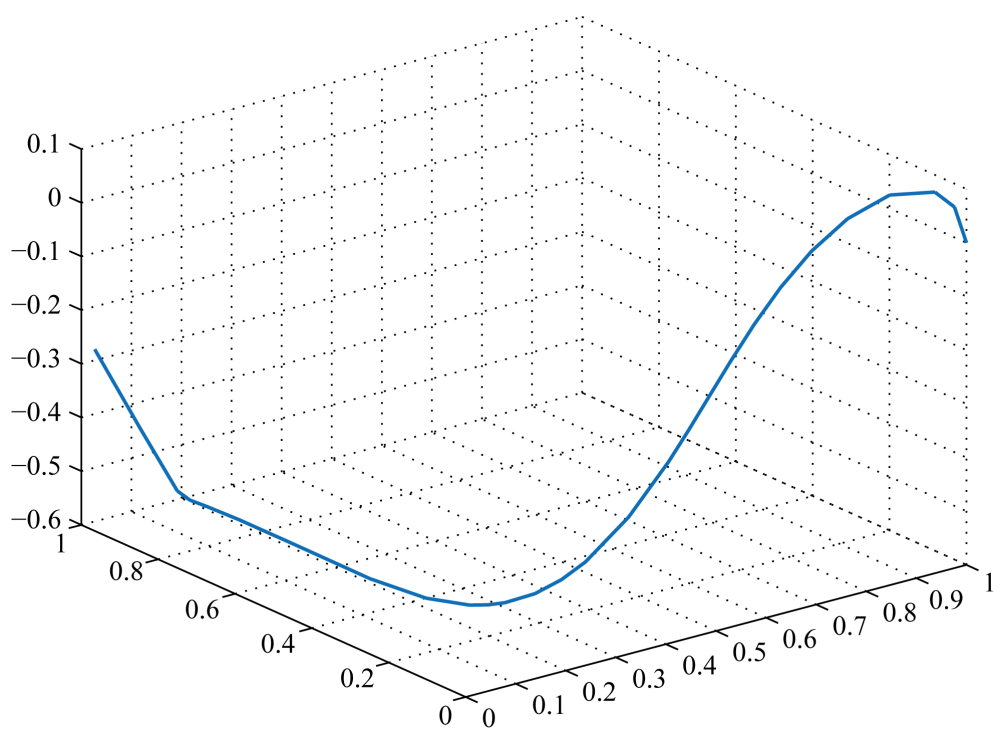

Figure 1. The optimal feedback control $\hat{u}$ in Example 1. 


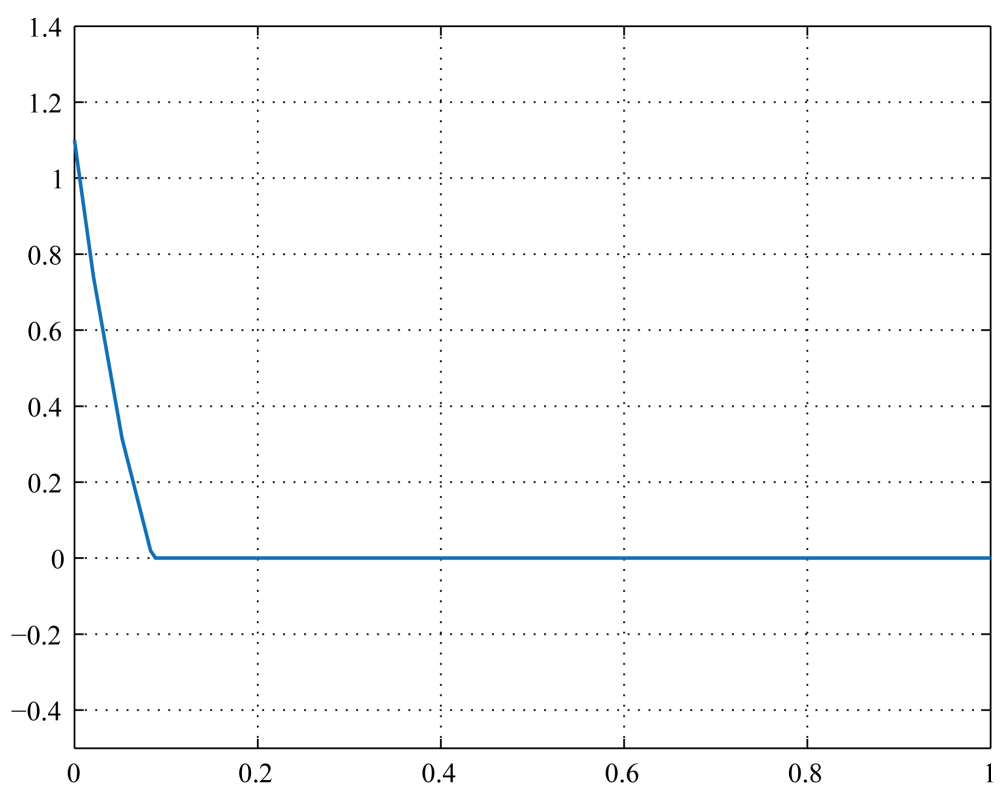

Figure 2. The dual variable $\rho^{\text {opt }}(\hat{\lambda})$ in Example 1.

\section{Box Constrained Optimal Control Problem}

In this section, we consider $U=\left\{u(t) \in \mathcal{R}^{m} \mid-1 \leq u_{i}(t) \leq 1, i=1,2, \cdots, m\right\}, \quad t \in\left[t_{0}, t_{f}\right]$, and $U$ is a unit box. Using the similar method in Section 2, the analytic solution to the box constrained optimal control problem $(\mathcal{P})$ can be derived.

\subsection{Global Optimization with Box Constraints}

Similarly, consider the general box constrained problem

$$
\min P(u) \text { s.t. } u \in U, U=\left\{-1 \leq u_{i} \leq 1, i=1,2, \cdots, m\right\},
$$

where $P(u)$ is assumed to be twice continuously differentiable in $\mathcal{R}^{m}$.

Denote

$$
\mathcal{S}=\left\{\rho \in \mathcal{R}^{m} \mid\left[\nabla^{2} P(u)+\operatorname{Diag}(\rho)\right]>\overrightarrow{0}, \rho \geq \overrightarrow{0}, \text { for every } u \in U\right\},
$$

where $\rho=\left(\rho_{1}, \rho_{2}, \cdots, \rho_{m}\right)^{\mathrm{T}}$ and $\operatorname{Diag}(\rho) \in \mathcal{R}^{m \times m}$ is a diagonal matrix with $\rho_{i}, i=1,2, \cdots, m$, being its diagonal elements. It is obvious that if $\hat{\rho} \in \mathcal{S}$, then $\rho \in \mathcal{S}$ for any $\rho \geq \hat{\rho}$. Parallel to what we did before, a differential flow $\hat{u}(\rho)$ is given as follow.

Assumed that $\rho^{*} \in \mathcal{S}$ and a nonzero vector $u^{*} \in U$ such that

$$
\nabla P\left(u^{*}\right)+\operatorname{Diag}\left(\rho^{*}\right) u^{*}=\overrightarrow{0},
$$

we focus on the flow $\hat{u}(\rho)$ which is well defined near $\rho^{*}$

$$
\left\{\begin{array}{l}
\mathrm{d} \hat{u}_{i}(\rho)=H_{i 1} \hat{u}_{1}(\rho) \mathrm{d} \rho_{1}+H_{i 2} \hat{u}_{2}(\rho) \mathrm{d} \rho_{2}+\cdots+H_{i m} \hat{u}_{m}(\rho) \mathrm{d} \rho_{m}, \\
\hat{u}_{i}\left(\rho^{*}\right)=u_{i}^{*}, \quad i=1,2, \cdots, m,
\end{array}\right.
$$

where $H(\rho, \hat{u}(\rho))=\left[H_{i j}(\rho)\right]_{m \times m}=-\left[\nabla^{2} P(\hat{u}(\rho))+\operatorname{Diag}(\rho)\right]^{-1}$ and $\hat{u}(\rho)=\left(\hat{u}_{1}(\rho), \hat{u}_{2}(\rho), \cdots, \hat{u}_{m}(\rho)\right)^{\mathrm{T}}$. Moreover, near $\rho^{*}$, the differential flow $\hat{u}(\rho)$ also satisfies

$$
\nabla P(\hat{u}(\rho))+\operatorname{Diag}(\rho) \hat{u}(\rho)=\overrightarrow{0} .
$$

Based on the extension theory, the solution $\hat{u}(\rho)$ of (34) can be extended to an interval in $\mathcal{S}$. Then, the 
canonical dual function is defined as follows

$$
P^{d}(\rho)=P(\hat{u}(\rho))+\sum_{i=1}^{m} \frac{\rho_{i}}{2}\left[\hat{u}_{i}^{2}(\rho)-1\right],
$$

and the canonical dual problem associated with the problem (32) can be formulated as follows

$$
\left(P^{d}\right): \max \left\{P^{d}(\rho)=P(\hat{u}(\rho))+\sum_{i=1}^{m} \frac{\rho_{i}}{2}\left[\hat{u}_{i}^{2}(\rho)-1\right] \mid \rho \in \mathcal{S}\right\} .
$$

Lemma 5. Let $\hat{u}(\rho)$ be a given flow defined by (33)-(34), and $P^{d}(\rho)$ be the corresponding canonical dual function defined by (36). Near $\rho^{*}$, we have

$$
\begin{gathered}
\nabla P^{d}(\rho)=\left(\frac{1}{2}\left[\hat{u}_{1}^{2}(\rho)-1\right], \frac{1}{2}\left[\hat{u}_{2}^{2}(\rho)-1\right], \cdots, \frac{1}{2}\left[\hat{u}_{m}^{2}(\rho)-1\right]\right)^{\mathrm{T}}, \\
\nabla^{2} P^{d}(\rho)=-\operatorname{Diag}(\hat{u}(\rho))\left[\nabla^{2} P(\hat{u}(\rho))+\operatorname{Diag}(\rho)\right]^{-1} \operatorname{Diag}(\hat{u}(\rho)) .
\end{gathered}
$$

Proof. Since $P^{d}(\rho)$ is differentiable, near $\rho^{*}$,

$$
\begin{aligned}
\frac{\partial P^{d}(\rho)}{\partial \rho_{i}} & =\nabla P(\hat{u}(\rho))^{\mathrm{T}} \frac{\partial \hat{u}(\rho)}{\partial \rho_{i}}+\frac{1}{2}\left[\hat{u}_{i}^{2}(\rho)-1\right]+\sum_{j=1}^{m} \rho_{j} \hat{u}_{j}(\rho) \frac{\partial \hat{u}_{j}(\rho)}{\partial \rho_{i}} \\
& =\nabla P(\hat{u}(\rho))^{\mathrm{T}} \frac{\partial \hat{u}(\rho)}{\partial \rho_{i}}+\rho^{\mathrm{T}} \operatorname{Diag}(\hat{u}(\rho)) \frac{\partial \hat{u}(\rho)}{\partial \rho_{i}}+\frac{1}{2}\left[\hat{u}_{i}^{2}(\rho)-1\right] \\
& =(\nabla P(\hat{u}(\rho))+\operatorname{Diag}(\rho) \hat{u}(\rho))^{\mathrm{T}} \frac{\partial \hat{u}(\rho)}{\partial \rho_{i}}+\frac{1}{2}\left[\hat{u}_{i}^{2}(\rho)-1\right] .
\end{aligned}
$$

By (35), it follows that $\frac{\partial P^{d}(\rho)}{\partial \rho_{i}}=\frac{1}{2}\left[\hat{u}_{i}^{2}(\rho)-1\right]$.

Form (34), we have $\frac{\partial \hat{u}_{i}(\rho)}{\partial \rho_{j}}=H_{i j}(\rho) \hat{u}_{j}(\rho)$, then

$$
\frac{\partial^{2} P^{d}(\rho)}{\partial \rho_{i} \partial \rho_{j}}=\hat{u}_{i}(\rho) \frac{\partial \hat{u}_{i}(\rho)}{\partial \rho_{j}}=\hat{u}_{i}(\rho) H_{i j}(\rho) \hat{u}_{j}(\rho) .
$$

By the definition of $H(\rho, \hat{u}(\rho))$, this concludes the proof of Lemma 5 .

Lemma 5 shows that the canonical dual function $P^{d}(\rho)$ is concave on $\mathcal{S}$, so, the problem $\left(P^{d}\right)$ can be solved by any commonly used nonlinear programming methods.

Theorem 4. (Perfect duality theorem) The canonical dual problem $\left(P^{d}\right)$ is perfectly dual to the primal problem (32) in the sense that if $\rho^{*} \in \mathcal{S}$ is a critical point of $P^{d}(\rho)$, then the vector $u^{*}=\hat{u}\left(\rho^{*}\right)$ is a KKT point of (32) and $P\left(u^{*}\right)=P^{d}\left(\rho^{*}\right)$.

Proof. By the KKT theory, $\rho^{*}$ is a KKT point of $\left(P^{d}\right)$ if and only if there exists one multiplier $\lambda \in \mathcal{R}^{m}$ such that

$$
\begin{gathered}
\nabla P^{d}\left(\rho^{*}\right)-\lambda=\overrightarrow{0}, \lambda^{\mathrm{T}} \rho^{*}=0, \lambda \leq \overrightarrow{0}, \rho^{*} \geq \overrightarrow{0}, \\
\nabla P^{d}\left(\rho^{*}\right)=\left(\frac{1}{2}\left[\hat{u}_{1}^{2}\left(\rho^{*}\right)-1\right], \frac{1}{2}\left[\hat{u}_{2}^{2}\left(\rho^{*}\right)-1\right], \cdots, \frac{1}{2}\left[\hat{u}_{m}^{2}\left(\rho^{*}\right)-1\right]\right)^{\mathrm{T}},
\end{gathered}
$$

where $\hat{u}(\rho)$ is defined as (33)-(34). This shows that $\hat{u}\left(\rho^{*}\right)$ is a KKT point of the primal problem (32). By the complementarity conditions (40), we have

$$
P^{d}\left(\rho^{*}\right)=P\left(\hat{u}\left(\rho^{*}\right)\right)+\sum_{i=1}^{m} \frac{\rho_{i}^{*}}{2}\left[\hat{u}_{i}^{2}\left(\rho^{*}\right)-1\right]=P\left(\hat{u}\left(\rho^{*}\right)\right)=P\left(u^{*}\right) .
$$

The proof is completed. 
Theorem 5. (Triality theorem) Consider $P(u)$ to be concave on the box $U$. If the flow $\hat{u}(\rho)$ defined by (33)-(35) meets a boundary point of $U$ at $\hat{\rho} \in \mathcal{S}$ such that $\hat{u}_{i}^{2}(\hat{\rho})=1, i=1,2, \cdots, m$, then $\hat{u}=\hat{u}(\hat{\rho})$ is a global minimizer of the problem (32). Further one has

$$
\min _{u \in U} P(u)=P(\hat{u}(\hat{\rho}))=P^{d}(\hat{\rho})=\max _{\rho \geq \hat{\rho}} P^{d}(\rho) .
$$

Proof. By Lemma 5 and the fact that $\hat{u}_{i}^{2}(\hat{\rho})=1, \hat{\rho} \in \mathcal{S}$, it can verify that $\nabla^{2} P^{d}(\rho) \leq 0$ and $\nabla P^{d}(\rho)$ is monotonously decreasing as $\rho \geq \hat{\rho}$. This means that $\hat{u}(\rho)$ will stay in $U$ and $P^{d}(\rho) \leq P^{d}(\hat{\rho})$ as $\rho \geq \hat{\rho}$. Using the definition of $\hat{u}(\rho)$ as well as $\mathcal{S}$, we have

$$
\begin{gathered}
\nabla\left\{P(\hat{u}(\rho))+\sum_{i=1}^{m} \frac{\rho_{i}}{2}\left[\hat{u}_{i}^{2}(\rho)-1\right]\right\}=\nabla P(\hat{u}(\rho))+\operatorname{Diag}(\rho) \hat{u}(\rho)=\overrightarrow{0}, \\
\nabla^{2}\left\{P(u)+\sum_{i=1}^{m} \frac{\rho_{i}}{2}\left(u_{i}^{2}-1\right)\right\}=\nabla^{2} P(u)+\operatorname{Diag}(\rho)>0, \forall u \in U .
\end{gathered}
$$

In the following deducing, we need to note the fact that since $P(u)$ is twice continuously differentiable on $\mathcal{R}^{m}$, there exists a positive real vector $\delta \in \mathcal{R}^{m}$ such that (42) holds in $\left\{u_{i}^{2}<1+\delta_{i}\right\}$ which contains $U$. So, we can show that $\hat{u}(\rho)$ is the global minimizer of $P(u)+\sum_{i=1}^{m} \frac{\rho_{i}}{2}\left(u_{i}^{2}-1\right)$ on $U$, and for any $\rho \geq \hat{\rho}$

$$
\begin{aligned}
P(u) & \geq P(u)+\sum_{i=1}^{m} \frac{\rho_{i}}{2}\left(u_{i}^{2}-1\right) \geq \inf _{U}\left\{P(u)+\sum_{i=1}^{m} \frac{\rho_{i}}{2}\left(u_{i}^{2}-1\right)\right\} \\
& =\left\{P(\hat{u}(\rho))+\sum_{i=1}^{m} \frac{\rho_{i}}{2}\left[\hat{u}_{i}^{2}(\rho)-1\right]\right\}=P^{d}(\rho) .
\end{aligned}
$$

Thus, we have

$$
P(u) \geq \max _{\rho \geq \hat{\rho}} P^{d}(\rho)=P^{d}(\hat{\rho})=P(\hat{u}(\hat{\rho}))+\sum_{i=1}^{m} \frac{\hat{\rho}_{i}}{2}\left[\hat{u}_{i}^{2}(\hat{\rho})-1\right]=P(\hat{u}(\hat{\rho})) .
$$

By (43), (44) and the canonical duality theory, it leads to the conclusion we desired.

\subsection{Analytic Solution to the Box Constrained Optimal Control Problem}

Now, let $P(u)=\frac{1}{2} u^{\mathrm{T}} R u+\hat{\lambda}^{\mathrm{T}}(t) B u$ in (32). For $\rho \in \mathcal{S}=\left\{\rho \in \mathcal{R}^{m} \mid \rho_{i} \in[0,+\infty), i=1,2, \cdots, m\right\}$ (since $R$ is a positive definite matrix), we define

$$
\hat{u}(\rho)=-[R+\operatorname{Diag}(\rho)]^{-1} B^{\mathrm{T}} \hat{\lambda}(t), \text { a.e. } t \in\left[t_{0}, t_{f}\right],
$$

and the canonical dual function

$$
P^{d}(\rho)=-\frac{1}{2} \hat{\lambda}^{\mathrm{T}}(t) B[R+\operatorname{Diag}(\rho)]^{-1} B^{\mathrm{T}} \hat{\lambda}(t)-\frac{1}{2} e^{\mathrm{T}} \rho .
$$

Set $y(\rho)=\hat{u}(\rho) \circ \hat{u}(\rho)=\left(\hat{u}_{1}^{2}(\rho), \hat{u}_{2}^{2}(\rho), \cdots, \hat{u}_{m}^{2}(\rho)\right)^{\mathrm{T}} \quad$ (the notation “。” denotes the Madamard product).

Lemma 6. Let $\hat{u}(\rho)$ be a given flow defined by (45), and $y(\rho)=\hat{u}(\rho) \circ \hat{u}(\rho) \cdot y_{i}(\rho)$ is monotonously decreasing with respect to $\rho_{i}$ on $[0,+\infty), i=1,2, \cdots, m$.

Proof. Notice that $y_{i}(\rho)=\hat{u}_{i}^{2}(\rho)$ and $\frac{\partial y_{i}(\rho)}{\partial \rho_{i}}=2 \hat{u}_{i}(\rho) \frac{\partial \hat{u}_{i}(\rho)}{\partial \rho_{i}}$. Let $H(\rho, \hat{u}(\rho))=-[R+\operatorname{Diag}(\rho)]^{-1}$ and $H_{i i}(\rho)$ be the $i^{\text {th }}$ diagonal element of $H$.

By properties of the positive definite matrix, it follows that the diagonal element $H_{i i}(\rho)$ is a negative real number which means that $\frac{\partial y_{i}(\rho)}{\partial \rho_{i}}=2 H_{i i}(\rho) \hat{u}_{i}^{2}(\rho) \leq 0$ because of the fact that $\frac{\partial \hat{u}_{i}(\rho)}{\partial \rho_{i}}=H_{i i}(\rho) \hat{u}_{i}(\rho)$. Thus, we can have the conclusion we desired. 
In the rest part of this section, we suppose that $R=\operatorname{Diag}(r)$ is a diagonal matrix with $r_{i}>0, i=1,2, \cdots, m$, being the diagonal elements. We have the following result.

Theorem 6. For the box constrained optimal control problem $(\mathcal{P})$, the analytic solution expressed by the co-state is given as follows

$$
\hat{u}=-\left[\frac{\left(B^{\mathrm{T}} \hat{\lambda}\right)_{1}}{\max \left[r_{1},\left|\left(B^{\mathrm{T}} \hat{\lambda}\right)_{1}\right|\right]}, \frac{\left(B^{\mathrm{T}} \hat{\lambda}\right)_{2}}{\max \left[r_{2},\left|\left(B^{\mathrm{T}} \hat{\lambda}\right)_{2}\right|\right]}, \cdots, \frac{\left(B^{\mathrm{T}} \hat{\lambda}\right)_{m}}{\max \left[r_{m},\left|\left(B^{\mathrm{T}} \hat{\lambda}\right)_{m}\right|\right]}\right]^{\mathrm{T}} .
$$

Proof. Set $B^{\mathrm{T}} \hat{\lambda}(t)=\left(\left(B^{\mathrm{T}} \hat{\lambda}(t)\right)_{1},\left(B^{\mathrm{T}} \hat{\lambda}(t)\right)_{2}, \cdots,\left(B^{\mathrm{T}} \hat{\lambda}(t)\right)_{m}\right)^{\mathrm{T}}$. It comes from Lemma 3.2 and (45) that $\hat{u}_{i}(\rho)=-\frac{\left(B^{\mathrm{T}} \hat{\lambda}(t)\right)_{i}}{r_{i}+\rho_{i}}, \quad y_{i}(\rho)=\frac{\left(B^{\mathrm{T}} \hat{\lambda}(t)\right)_{i}^{2}}{\left(r_{i}+\rho_{i}\right)^{2}}$ and $\frac{\partial y_{i}(\rho)}{\partial \rho_{i}}=-\frac{2\left(B^{\mathrm{T}} \hat{\lambda}(t)\right)_{i}^{2}}{\left(r_{i}+\rho_{i}\right)^{3}}, \frac{\partial y_{i}(\rho)}{\partial \rho_{j}}=0, i \neq j$. This means that $\hat{u}_{i}(\rho)$ and $y_{i}(\rho)$ depend only on the element $\rho_{i}$, i.e. $\hat{u}_{i}(\rho)=\hat{u}_{i}\left(\rho_{i}\right)$ and $y_{i}(\rho)=y_{i}\left(\rho_{i}\right)$.

Consider complementarity conditions $\rho_{i}\left(y_{i}(\rho)-1\right)=0, i=1,2, \cdots, m$. If $y_{i}(\rho)>1$ at the point $\rho_{i}=0$, by Lemma 6, it is easy to verify that there must exist one point $\hat{\rho}_{i}>0$ such that $\frac{\left(B^{\mathrm{T}} \hat{\lambda}(t)\right)_{i}^{2}}{\left(r_{i}+\hat{\rho}_{i}\right)^{2}}=1$. Otherwise, for any $\rho_{i} \geq 0$, we always have $y_{i}(\rho) \leq 1$. Thus, we define the vector $\rho^{\text {opt }} \in \mathcal{R}^{m}$,

$$
\rho_{i}^{\text {opt }}=\left\{\begin{array}{ll}
\left|\left(B^{\mathrm{T}} \hat{\lambda}(t)\right)_{i}\right|-r_{i} & \text { if }\left|\left(B^{\mathrm{T}} \hat{\lambda}(t)\right)_{i}\right|>r_{i}, \\
0 & \text { if }\left|\left(B^{\mathrm{T}} \hat{\lambda}(t)\right)_{i}\right| \leq r_{i},
\end{array} \text { a.e. } \quad t \in\left[t_{0}, t_{f}\right],\right.
$$

which can be rewritten as $\rho_{i}^{\text {opt }}=\max \left[0,\left|\left(B^{\mathrm{T}} \hat{\lambda}(t)\right)_{i}\right|-r_{i}\right]$, a.e. $t \in\left[t_{0}, t_{f}\right]$. It follows form (45) and (48) that a.e. $t \in\left[t_{0}, t_{f}\right]$,

$$
\hat{u}_{i}\left(\rho^{\text {opt }}\right)=-\frac{\left(B^{\mathrm{T}} \hat{\lambda}(t)\right)_{i}}{\max \left[r_{i},\left|\left(B^{\mathrm{T}} \hat{\lambda}(t)\right)_{i}\right|\right]}, \quad i=1,2, \cdots, m .
$$

In what follows, parallel to the proof of Theorem 2, we shall show that $\hat{u}\left(\rho^{\text {opt }}\right)$ is the analytic solution for the problem $(\mathcal{P})$.

By statements as the above and Lemma 6, we have $\hat{u}(\rho) \in U$ for any $\rho \geq \rho^{\text {opt }}$, and the function family $\left\{f_{\rho}(u)\right\}$ is given as follows

$$
f_{\rho}(u)=P(u)+\sum_{i=1}^{m} \frac{\rho_{i}}{2}\left(u_{i}^{2}-1\right)=\frac{1}{2} u^{\mathrm{T}} R u+\hat{\lambda}^{\mathrm{T}}(t) B u+\sum_{i=1}^{m} \frac{\rho_{i}}{2}\left(u_{i}^{2}-1\right),
$$

where $\rho$ is a parameter. Using (45) and (49), it is obvious that $\hat{u}(\rho)$ is a global minimizer of the problem $\min f_{\rho}(u)$ on $U$ by the fact that $\nabla f_{\rho}(\hat{u}(\rho))=0$ and $\nabla^{2} f_{\rho}(u)>0, \forall u \in U$. Further, we have

$$
P(u) \geq f_{\rho}(u) \geq \inf _{U} f_{\rho}(u)=f_{\rho}\left(\hat{u}\left(\rho^{o p t}\right)\right)=P^{d}\left(\rho^{o p t}\right) .
$$

By Lemma 5 and (46), we have

$$
P(u) \geq \max _{\rho \geq \rho^{o p t}} P^{d}(\rho)=P^{d}\left(\rho^{\text {opt }}\right)=P\left(\hat{u}\left(\rho^{\text {opt }}\right)\right) .
$$

Thus, $\hat{u}\left(\rho^{\text {opt }}\right)$ is a global minimizer of the problem (7). Consider $\rho^{\text {opt }}$ as a function with respect to the co-state $\hat{\lambda}$, by Lemma 1 , then $\hat{u}$ expressed by (47) is the analytic solution for the optimal control problem $(\mathcal{P})$. This completes the proof. 


\subsection{Applications}

We will give an example to illustrate our results.

Example 2: For the box constrained optimal control problem $(\mathcal{P})$, we consider $A=\left(\begin{array}{cc}2 & 7 \\ -2.5 & 3\end{array}\right)$, $B=\left(\begin{array}{cc}2 & -5 \\ 1.5 & 7\end{array}\right), \quad Q=\left(\begin{array}{cc}2.4 & 3 \\ -2 & -1\end{array}\right), \quad R=\left(\begin{array}{ll}2 & 0 \\ 0 & 3\end{array}\right), x(0)=(1,1)^{\mathrm{T}}, t_{0}=0$, and $t_{f}=1$, where $Q \geq 0, R>0$ satisfying the assumption in (1).

Following idea of Lemma 1 and Theorem 2 as above, we need to solve a system on the state and co-state for deriving the optimal solution

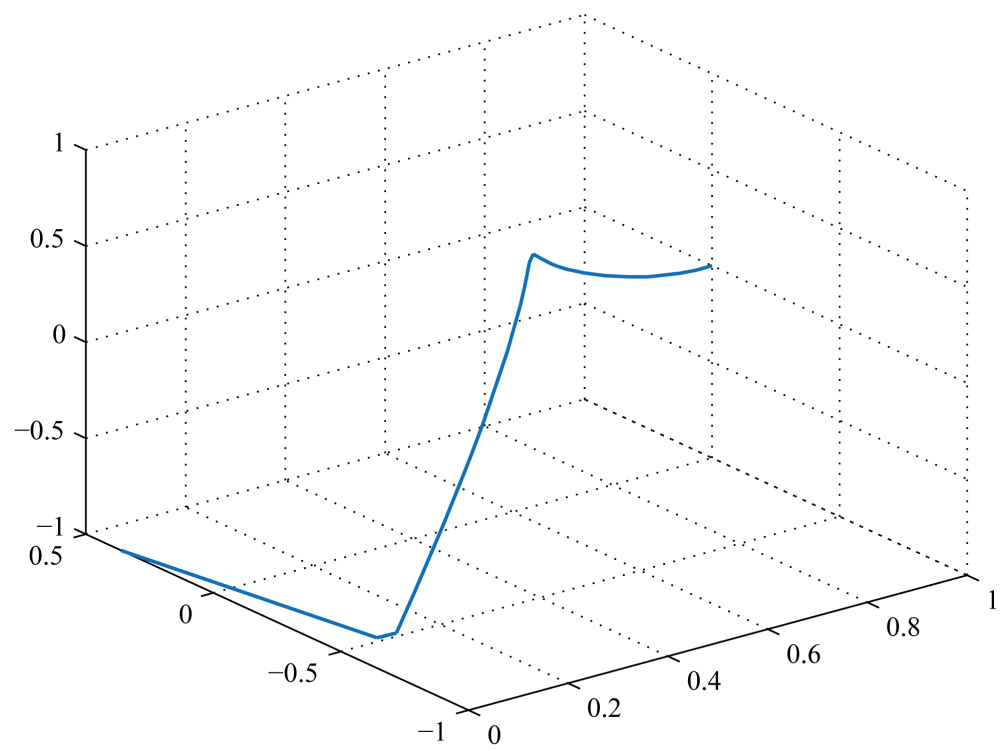

Figure 3. The optimal feedback control $\hat{u}$ in Example 2 .

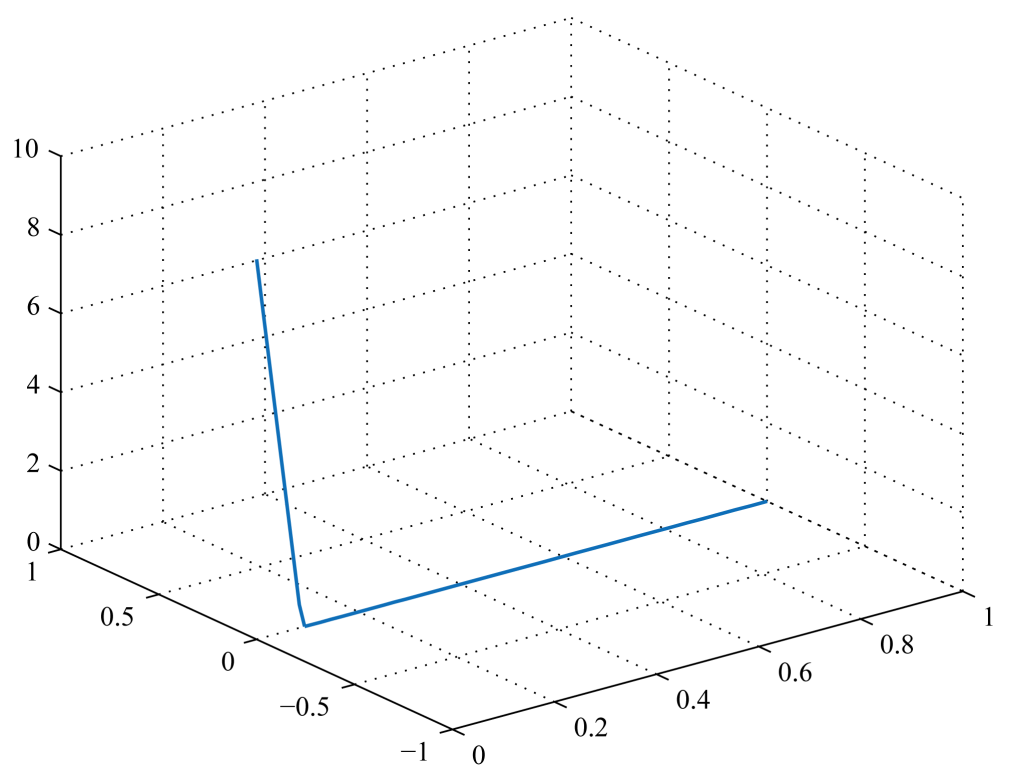

Figure 4. The dual variable $\rho^{\text {opt }}(\hat{\lambda})$ in Example 2. 


$$
\begin{gathered}
\dot{\hat{x}}=H_{\lambda}(t, \hat{x}, \hat{u}, \hat{\lambda})=A \hat{x}+B \hat{u}, \hat{x}(0)=(1,1)^{\mathrm{T}}, \\
\dot{\hat{\lambda}}=-H_{x}(t, \hat{x}, \hat{u}, \hat{\lambda})=-A^{\mathrm{T}} \hat{\lambda}-Q \hat{x}, \hat{\lambda}(1)=\overrightarrow{0},
\end{gathered}
$$

and

$$
\hat{u}_{1}(t)=-\frac{\left(B^{\mathrm{T}} \hat{\lambda}(t)\right)_{1}}{\max \left[2,\left(B^{\mathrm{T}} \hat{\lambda}(t)\right)_{1} \mid\right]}, \hat{u}_{2}(t)=-\frac{\left(B^{\mathrm{T}} \hat{\lambda}(t)\right)_{2}}{\max \left[3,\left(B^{\mathrm{T}} \hat{\lambda}(t)\right)_{2} \mid\right]}, \text { a.e. } t \in[0,1] .
$$

By solving Equations (52)-(54) in MATLAB, we can obtain the optimal optimal feedback control $\hat{u}$ and the dual variable $\rho^{\text {opt }}(\hat{\lambda})$ as follows (see Figure 3, Figure 4).

\section{Acknowledgements}

We thank the Editor and the referee for their comments. Research of D. Wu is supported by the National Science Foundation of China under grants No.11426091, 11471102.

\section{References}

[1] Casti, J. (1980) The Linear-Quadratic Control Problem: Some Recent Results and Outstanding Problems. SIAM Review, 22, 459-485. http://dx.doi.org/10.1137/1022089

[2] Robinson, C. (1995) Dynamical Systems. CRC Press, London.

[3] Anderson, B.D.O. and Moore, J.B. (1971) Linear Optimal Control. Prentice-Hall, New Jersey.

[4] Heinkenschloss, M. and Tröltzsch. F. (1999) Analysis of the Lagrange-SQP-Newton Method for the Control of a Phase Field Equation. Control and Cybernetics, 28, 177-211.

[5] Kunisch, K. and Sachs, E.W. (1992) Reduced SQP Methods for Parameter Identification Problems. SIAM Journal on Numerical Analysis, 29, 1793-1820. http://dx.doi.org/10.1137/0729100

[6] Tröltzsch, F. (1994) An SQP-Method for Optimal Control of a Nonlinear Heat Equation. Control and Cybernetics, 23, 268-288.

[7] Tian, T. and Dunn, J.C. (1994) On the Gradient Projection Method for Optimal Control Problems with Nonnegative $L^{2}$ inputs. SIAM Journal on Control and Optimization, 32, 516-537.

[8] Kelley, C.T. and Sachs, E.W. (1995) Solution of Optimal Control Problems by a Pointwise Projected Newton Method. SIAM Journal on Control and Optimization, 33, 1731-1757. http://dx.doi.org/10.1137/S0363012993249900

[9] Gao, D.Y., Ruan, N. and Latorre, V. (2014) Canonical Duality-Triality Theory: Bridge between Nonconvex Analysis/ Mechanics and Global Optimization in Complex Systems. Mathematics and Mechanics of Solids, 12, 716-735.

[10] Gao, D.Y. and Ruan, N. (2015) Canonical Duality Theory for Solving Nonconvex/Discrete Constrained Global Optimization Problems. Mathematics and Mechanics of Solids. http://dx.doi.org/10.1177/1081286515591087

[11] Latorre, V. and Sagratella, S. (2014) A Canonical Duality Approach for the Solution of Affine Quasi-Variational Inequalities. Journal of Global Optimization, 1, 1-17.

[12] Gao, D.Y. and Ruan, N. (2015) Application of Canonical Duality Theory to Fixed Point Problem. Springer Proceedings in Mathematics \& Statistics, 95, 157-163. http://dx.doi.org/10.1007/978-3-319-08377-3_17

[13] Zhu, J., Tao, S. and Gao, D.Y. (2009) A Study on Concave Optimization via Canonical Dual Function. Journal of Computational and Applied Mathematics, 224, 459-464. http://dx.doi.org/10.1016/j.cam.2008.05.011

[14] Zhu, J. and Yan, W. (2009) Solution to Constrained Nonlinear Programming by Canonical Dual Method. Lecture Notes on Decision Sciences, 12, 217-222.

[15] Zhu, J., Wu, D. and Gao, D.Y. (2012) Applying the Canonical Dual Theory in Optimal Control Problems. Journal of Global Optimization, 29, 377-399. http://dx.doi.org/10.1007/s10898-009-9474-3

[16] Adjiman, C.S., Androulakis, I.P. and Floudas, C.A. (1998) A Global Optimization Method, $\alpha$ BB, for General TwiceDifferentiable Constrained NLPs-II. Implementation and Computational Results. Computers \& Chemical Engineering, 22, 1159-1179. http://dx.doi.org/10.1016/S0098-1354(98)00218-X

[17] Adjiman, C.S., Androulakis, I.P. and Floudas, C.A. (1998) A Global Optimization Method, $\alpha$ BB, for General TwiceDifferentiable Constrained NLPs-I. Theoretical Advances. Computers \& Chemical Engineering, 22, 1137-1158. http://dx.doi.org/10.1016/S0098-1354(98)00027-1 
[18] Keller, H.B. (1976) Numerical Solution of Two Point Boundary Value Problems. SIAM, Philadelphia. http://dx.doi.org/10.1137/1.9781611970449

[19] Ascher, U.M., Mattheij, R.M.M. and Russell, R.D. (1995) Numerical Solution of Boundary Value Problems for Ordinary Differential Equations (Classics in Applied Mathematics). SIAM, Philadelphia.

http://dx.doi.org/10.1137/1.9781611971231 